\title{
ROLE OF MRI T2* IMAGING IN EVALUATION OF LIVER AND CARDIAC IRON OVERLOAD, ITS CORRELATION WITH SERUM FERRITIN AND CARDIAC 2D ECHO CORRELATION
}

\author{
Sanjiv Patel ${ }^{1}$, Sapna Patel2 ${ }^{2}$ Hardik Jadav³, Kushal Makwana ${ }^{4}$
}

${ }^{1}$ Assistant Professor, Department of Radiodiagnosis, GCRI, Ahmedabad.

${ }^{2}$ Assistant Professor, Department of Radiodiagnosis, GCRI, Ahmedabad.

$3^{3}$ rd Year Resident, Department of Radiodiagnosis, GCRI, Ahmedabad.

${ }^{4} 2^{\text {nd }}$ Year Resident, Department of Radiodiagnosis, GCRI, Ahmedabad.

\section{BACKGROUND}

ABSTRACT

Patients with chronic haemolytic anaemia like thalassemia and sickle cell anaemia need repeated blood transfusions, which lead to iron overload and cellular damage, especially in the heart and liver. Classically, serum ferritin and liver biopsy have been used to monitor patient's response to chelation therapy. Magnetic resonance imaging (MRI) has been proved effective in detecting and quantifying iron in the heart and liver.

The aim of this study is to find out the correlation between the quantification of cardiac and liver iron overload in repeatedly transfused patients using MRI T2* imaging with serum ferritin and cardiac output using 2D echocardiography respectively.

\section{MATERIALS AND METHODS}

In 400 cases of repeatedly transfused patients, hepatic and myocardial iron overload was measured by multi-breath holds MRI T2* and compared to serum ferritin (a traditional marker of iron overload).

\section{RESULTS}

1. Moderate positive correlation was observed between Cardiac T2* measurements and 2D echo (ejection fraction) (P $<0.0001$, $r=0.39$ ).

2. Moderate positive correlation was observed between serum ferritin and liver T2* MRI $(\mathrm{P}<0.0001, \mathrm{r}=0.41)$.

\section{CONCLUSION}

Routine evaluation of liver and heart iron content using MRI T2* is suggested to better evaluate the haemosiderosis status in patients with chronic haemolytic anaemia. Also, regular follow-up studies can alleviate the need for repeated liver biopsy and detect cardiac iron overload before starting of cardiac dysfunction.

\section{KEYWORDS}

Thalassemia, Haemosiderosis, MR Evaluation.

HOW TO CITE THIS ARTICLE: Patel S, Patel S, Jadav H, et al. Role of MRI T2* imaging in evaluation of liver and cardiac iron overload, its correlation with serum ferritin and cardiac 2D echo correlation. J. Evolution Med. Dent. Sci. 2018;7(04):462-466, DOI: $10.14260 /$ jemds/2018/103

\section{BACKGROUND}

Patients with thalassemia and some patients with sickle cell disease (SCD) patients need regular blood transfusions to survive beyond the second decade of life.(1) Although, transfusions improve health and survival, the chronic administration of large amounts of blood combined with extravascular haemolysis and an increase in the intestinal absorption of iron inevitably leads - despite chelation therapy - to significant haemosiderosis of all organs.(2) Iron is an essential element that forms an important component of metabolic and biological processes, but when present in excess it can produce tissue damage due to oxidative stress. (3) Excess body iron may accumulate in the liver, spleen, heart, bone marrow, pituitary, pancreas and the central nervous system causing damage to these organs.(4)

'Financial or Other Competing Interest': None.

Submission 04-09-2017, Peer Review 05-01-2018,

Acceptance 12-01-2018, Published 22-01-2018.

Corresponding Author:

Dr. Sapna Patel,

GCRI, Civil Campus,

Aswarva,

Ahmedabad.

E-mail: drsapnapatel@gmail.com

DOI: $10.14260 /$ jemds $/ 2018 / 103$
Iron overload cardiomyopathy (IOC) results from the accumulation of iron in the myocardium and it often occurs late and can be hard to reverse once established and it is the leading cause of death in patients receiving chronic blood transfusion therapy. $(5,6)$ Unfortunately, significant cardiac iron burden can occur despite low liver iron concentration. ${ }^{(7)}$ There is an important clinical need to reliably risk stratify patients for the development of heart failure so that chelation therapy can be intensified and cardiac morbidity averted.(8) Indirect markers of current cardiac iron overloading, namely single measurements of serum ferritin and biopsy determined liver iron concentration are inadequate for identifying myocardial iron deposition in the chronically transfused patients. $(9)$

Ferritin is a metalloprotein that are found in cells. It stores and releases iron in a controlled manner. In normal individuals, a small amount appears in the circulation and in general reflects the total body iron. Normal values of serum ferritin for men and women are $12-300 \mathrm{ng} / \mathrm{mL}$ and $12-150$ $\mathrm{ng} / \mathrm{mL}$, respectively.(10) It is an acute phase reactant and the serum levels may be disproportionately greater than the degree of iron loading in infections, inflammatory states, liver dysfunction and malignancies.(10) 
However, magnetic resonance can exploit the paramagnetic properties of tissue iron to provide direct, noninvasive iron assessments, and clinically this has been achieved by assessing the $\mathrm{T} 2$ and $\mathrm{T} 2 *$ relaxation parameters.(11) Magnetic resonance does not image the iron directly, but instead images water protons as they diffuse near iron deposits in the tissue of interest.(12) The iron acts as small magnets, destroying the homogeneity of the magnetic field in iron-laden tissues. The moving water protons experience significantly different magnetic profiles and became desynchronised from one another. This causes the image to darken at a rate proportional to the iron concentration. ${ }^{(3)}$

This technique is simple, allowing rapid and reproducible quantification of myocardial and hepatic iron and therefore allows pre-symptomatic detection of myocardial siderosis.(13) The $2^{*}$ technique has the potential to become a non-invasive standard technique in assessing tissue iron.(14) In our study, we compared the MRI T2* results with serum ferritin levels of patients on regular blood transfusion and correlated with ejection fraction on 2D echo.

\section{MATERIALS AND METHODS}

\section{Study Type}

Diagnostic centre based cross-sectional study.

\section{Study Period}

Since April 2012.

\section{Sample Size}

Patients attending hospital during study period- 400 cases.

\section{Sampling Method}

Convenient sampling.

\section{Statistical Methods}

Pearson's tests were applied wherever applicable. Statistical software- Epi-Info 7.0 was used for computations.

Since April 2012, a cross-sectional study of patients referred for MRI assessment of cardiac and liver siderosis at Dr. Narendra L Patel X-Ray House, SRL Diagnostic Centre, Ahmedabad has undergone a standardised imaging protocol which includes measurement of cardiac and liver T2*.

We studied a total of 400 patients, out of which 117 are follow-up patients, 283 patients with B-thalassemia (87 females and 197 males) with a median age of $18.67 \pm 9.34$ (range: 3 - 62 years). The exclusion criteria were as follows: Patients younger than 3 years old, as they did not receive enough transfusion to develop hepatic or myocardial siderosis and patients with associated hepatitis B or C to avoid any other parenchymal changes that could alter our results. Our patients did not have history of other congenital or acquired liver disease. All patients were on regular transfusion program and on iron chelation therapy for at least 18 months.

\section{The Present Study considers the following Parameters-}

(a) Serum ferritin, (b) Liver iron concentration, (c) Myocardial iron concentration, (d) Ejection fraction.

\section{*MRI Technique}

Magnetic resonance imaging studies were performed using a 1.5-T MAGNETOM Avanto System (Siemens Medical Systems). For the liver, three transverse images were recorded using a body matrix coil, visualising the right liver lobe (L) and posterior vertebral muscles (M) in the same slice. In order to measure the $\mathrm{T} 2 *$ relaxation Gradient echo sequence (GRE T2*), sequence with 12 different echo times (1.2 - $21.8 \mathrm{~ms}$ ) was used to acquire images. The field of view was 250 - $450 \mathrm{~mm}$ (depends on the patient's body circumference) and the matrix was $128 \times 192$. In order to reduce the scan time with phase, FOV is reduced to $70 \%$. Three images were obtained at the middle of the liver with each slice acquired with the patient instructed to hold their breath. TR was $200 \mathrm{~ms}$, flip angle was $20^{\circ}$ and slice thickness was $8 \mathrm{~mm}$. Acquisition time per slice was $10-15 \mathrm{~s}$. For the heart, three mid-ventricular short axis slice was imaged with a slice thickness of $6 \mathrm{~mm}$ and a slice gap of $0.6 \mathrm{~mm}$. Using body matrix coil, gradient echo sequence (T2*) with eight echo times (3.6 - $16 \mathrm{~ms}$ ) was used to obtain the images. The field of view was $280-320 \mathrm{~mm}$ with the phase FOV was between $60 \%$ and $80 \%$. With conventional white blood technique, prospective ECG triggering was used. The image was acquired at the end diastolic phase where the heart motion was minimal. The TR between each radiofrequency was $20 \mathrm{~ms}$. The matrix was $128 \times 192$. Acquisition time per slice was 8 - 12. Total scanning for the liver and heart T2* assessment was completed within 10 mins.

This includes the setup time for ECG, localiser scan and 6 breath holds (three for the liver and three for the heart). For analysis, the signal intensity of a full-thickness region of the left ventricular septum was measured for each echo time using Thalassemia tools (a plug-in of CMR tools, Cardiovascular Imaging Solutions, London, UK). Correction for positional changes between breath-holds was made to ensure regions of interest were within the myocardium. The accuracy of the $\mathrm{T} 2 *$ imaging is limited to the septum due to susceptibility effect artifacts from anterior and posterior cardiac veins and lungs that contaminate images of the other $\mathrm{LV}$ regional walls.

To derive $\mathrm{T} 2 *$, a mono-exponential trendline was fitted with an equation in the form of $\mathrm{y}=\mathrm{Ke} \_\mathrm{TE} / \mathrm{T} 2 *$ where $\mathrm{K}(1086$ HS Fahmy et al) represents a constant, TE represents the echo time and $y$ represents the image signal intensity. Analysis of the heart and liver T2* can be completed within 2 mins. In heavily-iron loaded tissues, signal decay occurs rapidly with noise dominating the signal at later echo times.

To minimise the potential for noise to cause overestimation of $\mathrm{T} 2 *$ in heavily iron-loaded tissues, these later points are not used for curve fitting for very low values of T2* and we did truncation as He et al (11). For the measurement of liver T2*, a single transaxial slice through the centre of the liver was imaged at 8 echo times ( $2.3-16 \mathrm{~ms})$. The TR was set to $200 \mathrm{~ms}$ without cardiac gating.

Signal intensity analysis was performed in the periphery of the liver away from the large central vessels. 

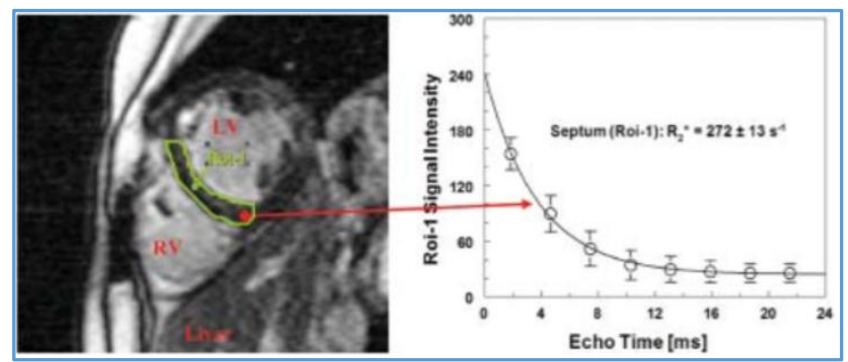

Image 1. Shows Technique of Measurement to Determine the Liver and Cardiac Iron Concentration using MRI T2* Images

Image 1

Mid-papillary short-axis slice at an echo time of $7.5 \mathrm{~ms}$ in a patient with thalassemia major for assessing the signal intensities in the septum (ROI-1) (single-breath-hold multiecho method $\mathrm{Te}=1.9-22$ ms: $1.5 \mathrm{~T}$ Siemens Magnetom Symphony, UKE Hamburg). The subsequent exponential fit yields a short relaxation time $\mathrm{T} 2 *=1000 / \mathrm{R} 2 *=3.7 \mathrm{~ms}$, while the liver iron concentration in this patient is relatively low LIC $(B L S)=1090+/-114 \mu \mathrm{g} / \mathrm{g}$ liver*

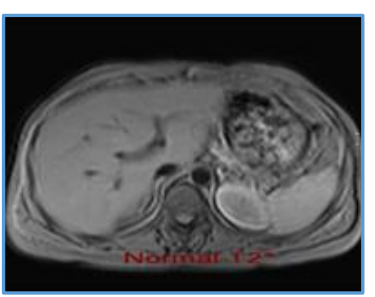

$2 a$

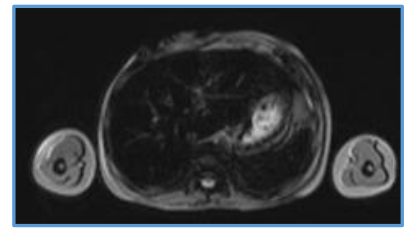

$2 c$

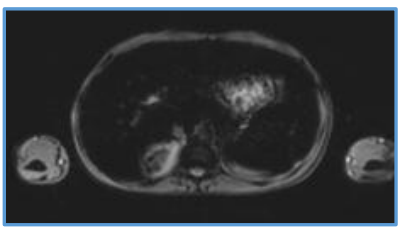

2d

Image 2. Shows Grades of Liver Iron Concentration using

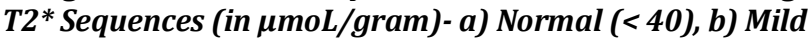
(55), c) Moderate (220), d) Severe (370)

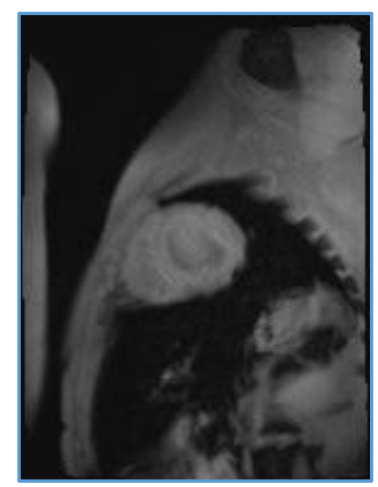

2a

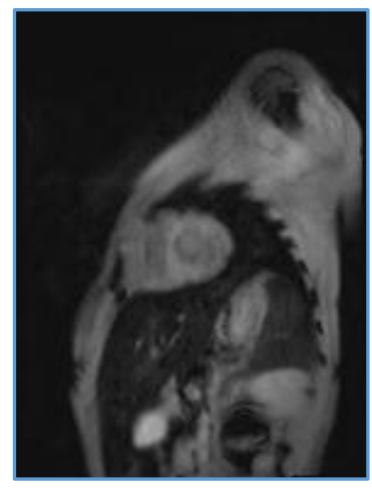

2b

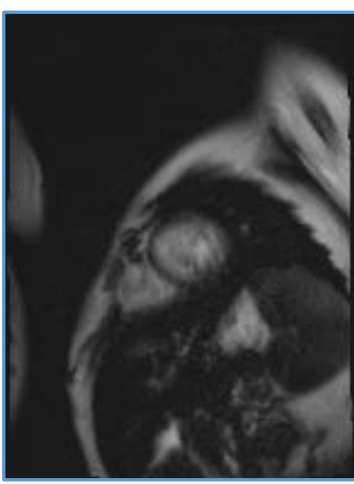

2c 2d

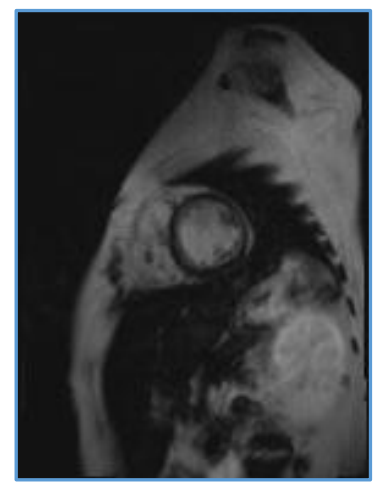

Image 3. Shows Grades of Cardiac Iron Concentration using T2* Sequences (in ms), a) Normal (29.82), b) Mild (19.8), c) Moderate (8.16), d) Severe (6.36)

\begin{tabular}{|c|c|}
\hline Liver Iron Concentration & LIC $(\boldsymbol{\mu m o L} / \mathbf{g})$ \\
\hline Normal & Less than 40 \\
\hline Mild & $40-100$ \\
\hline Moderate & $100-200$ \\
\hline Severe & Above 300 \\
\hline Cut-Off Points in this MRI Instrument are as Follows \\
\hline
\end{tabular}

\begin{tabular}{|c|c|}
\hline Myocardial Loading & Myocardial T2 (ms) \\
\hline Normal & More than 20 \\
\hline Mild & $12-20$ \\
\hline Moderate & $8-12$ \\
\hline Severe & Less than 8 \\
\hline Cut-Off Points for Cardiac Iron Overload \\
\hline
\end{tabular}

Guidelines for Iron Assessment

- Patients and serum ferritin levels.

- Measurements were carried out by a micro-particle enzyme immunoassay technique.

\section{Statistical Analysis}

Summary data are presented as mean and standard deviation. For reproducibility data the coefficient of variation was defined as the standard deviation of the differences between the two separate measurements, divided by their mean and expressed as a percentage. T2* values measured in healthy volunteers showed a normal distribution and are expressed with $95 \%$ reference ranges.

Pearson's coefficient of correlation was used to assess the degree of association between myocardial T2* and liver T2* and myocardial $\mathrm{T} 2 *$ and $2 \mathrm{D}$ echo (ejection fraction).

\section{RESULTS}

Pearson's coefficient $=0.39$ for correlation between Cardiac iron and Cardiac output, which implies-

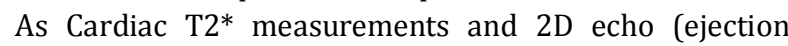
fraction) were positively skewed, Pearson's tests gave a correlation coefficient of 0.39 which is moderately positive significantly $(\mathrm{P}<0.0001)$. It suggests there is decrease in cardiac output with increase in loading of cardiac iron (Fig. 1.2). The main characteristics of these patients are summarised in Table 1. 


\begin{tabular}{|c|c|c|}
\hline \multirow{2}{*}{ Characteristics } & Patients N= 400 \\
\cline { 2 - 3 } & No. & \% \\
\hline \multicolumn{3}{|c|}{ Age (Years) } \\
\hline $10-30$ & 35 & 8.75 \\
\hline$>/=30$ & 345 & 86.25 \\
\hline \multicolumn{3}{|c|}{ Min - Max= 5-62 } \\
\hline \multicolumn{3}{|c|}{ Mean + SD=18.67+/-7.76 } \\
\hline \multicolumn{3}{|c|}{ Gender } \\
\hline Male & 280 & 70 \\
\hline Female & 120 & 30 \\
\hline Table 1. The Socio-Demographic Characteristic \\
of 400 Patients \\
\hline
\end{tabular}

Pearson's coefficient $=0.41$ for correlation between Serum ferritin and Liver iron load, which implies-

There was a significant (moderate positive) correlation between liver $\mathrm{T} 2^{*}$ and serum ferritin levels noted (Fig. 1.1). Pearson's test gave a correlation coefficient of 0.41 , which is moderately positive significantly $(p<0.0001)$. Liver iron rises steadily with increase in serum ferritin levels.

\section{Normal T2* Values}

The normal values for $\mathrm{T}^{*}$ using the technique described above were: Heart 5216 ms, liver 337 ms, skeletal muscle 305 ms and spleen $5622 \mathrm{~ms}$.

The distribution of hepatic and cardiac MRI T2* is shown in Tables 2.

\begin{tabular}{|c|c|c|}
\hline \multirow{2}{*}{ Characteristics } & Patients N = 400 \\
\cline { 2 - 3 } & No. & \%yocardial T2* \\
\hline Normal & 229 & 57.25 \\
\hline Mild & 34 & 8.5 \\
\hline Moderate & 61 & 15.25 \\
\hline Severe Min - Max= 4.73-100 \\
\hline \multicolumn{3}{|c|}{ Mean +/-SD=23.57+/-14.40 } \\
\hline \multicolumn{3}{|c|}{ Liver T2* } \\
\hline Normal & 8 & 2 \\
\hline Mild & 32 & 8 \\
\hline >/= Mild - & 37 & 9.25 \\
Moderate/Moderate & 323 & 80.75 \\
\hline $\begin{array}{c}\text { >/= Moderate - } \\
\text { Severe/Severe }\end{array}$ & Min - Max= 15-370 \\
\hline \multicolumn{2}{|c|}{ Mean +/- SD= 301+/-96.42 } \\
\hline \multicolumn{2}{|c|}{ Table 2. The Distribution between Myocardial and Liver } \\
T2* Values \\
\hline
\end{tabular}

\section{DISCUSSION}

\section{Iron Overload Pathophysiology}

Iron overload occurs either due to excess gastrointestinal absorption or secondary to repeated blood transfusions. The human body has no mechanism for excreting excess iron, which is stored as crystalline iron oxide within ferritin and hemosiderin in the body. The aetiology of the iron overload effects the tissue distribution of iron. In hereditary haemochromatosis, iron is carried from the intestine to the liver via the portal vein (as transferrin) and deposited in the periportal hepatocytes. In severe disease, iron is also deposited in the pancreas, heart and endocrine organs. In thalassemia, iron overload results from both excessive iron absorption and transfusional siderosis. Transfusional iron leads to iron deposition in the reticuloendothelial system of the spleen, liver and bone marrow. In advanced cases iron also accumulates in parenchymal cells of the liver, heart, pancreas and endocrine organs, which are sensitive to the toxic effects of iron. When the iron-binding capacity of transferrin is exhausted, free iron appears as non-transferrin bound iron (NTBI). The toxicity of NTBI is much higher than bound iron and promotes hydroxyl-radical formation resulting in peroxidative damage to membrane lipids and proteins. In the heart, this results in impaired function of the mitochondrial respiratory chain and is manifested clinically as heart failure. As iron accumulates in the normal storage form in the heart the T2* falls, but there is little effect on cardiac function until a threshold is reached where the iron storage capacity is exhausted. At this point NTBI starts to appear, which profoundly affects cardiac function. Thus, the relationship between the measured $\mathrm{T} 2 *$ and cardiac function is shallow until a critical level is reached, after which rapid deterioration occurs. This explains why identification of abnormal systolic function is a late sign of iron toxicity. Iron clears more slowly from the heart than the liver, which may contribute to the high mortality of patients with established cardiomyopathy despite intensive chelation. Using this $\mathrm{T} 2 *$ technique, it is possible to identify much earlier those patients who require intensive chelation prior to the onset of systolic dysfunction and this should avoid the mortality associated with overt heart failure.

\section{CONCLUSION}

MRI T2* evaluation of liver and heart iron content helps to determine haemosiderosis status in patients with chronic haemolytic anaemia. Regular follow-up with MRI T2* imaging can be useful in avoiding unnecessary repeated liver biopsy. It can even detect cardiac iron overload before cardiac dysfunction.

\section{REFERENCES}

[1] Borgna-Pignatti C, Rugolloto S, De Stefano P, et al. Survival and complications in patients with thalassemia major treated with transfusion and deferoxamine. Haematologica 2004;89(10):1187-93.

[2] Mavrogeni SI, Maris T, Gouliamos A, et al. Myocardial iron deposition in beta-thalassemia studied by magnetic resonance imaging. Int J Cardiac Imaging 1998;14(2):117-22.

[3] Liu P, Olivieri N. Iron overload cardiomyopathies: new insights into an old disease. Cardiovasc Drugs Ther 1994;8(1):101-10.

[4] Gujja P, Rosing DR, Tripodi DJ, et al. Iron overload cardiomyopathy: better understanding of an increasing disorder. J Am Coll Cardiol 2010;56(13):1001-12.

[5] Buja LM, Roberts WC. Iron in the heart. Etiology and clinical significance. Am J Med 1971;51(2):209-21.

[6] Olivieri NF, Nathan DG, MacMillan JH, et al. Survival in medically treated patients with homozygous Bthalassemia. N Engl J Med 1994;331(9):574-8.

[7] Fischer R, Longo F, Nielsen P, et al. Monitoring long term efficacy of iron chelation therapy by deferiprone and desferrioxamine in patients with beta-thalassemia major: application SQUID biomagnetic liver susceptometry. Br J Haematol 2003;121(6):938-48. 


\section{Jemds.com}

[8] Roghi A, Cappellini MD, Wood JC, et al. Absence of cardiac siderosis despite hepatic iron overload in Italian patients with thalassemia intermedia: an MRI T2* study. Ann Hematol 2010;89(6):585-9.

[9] Wood JC, Tyszka JM, Carson S, et al. Myocardial iron loading in transfusion-dependent thalassemia and sickle cell disease. Blood 2004;103(5):1934-6.

[10] Azarkeivan A, Hashemieh M, Akhlaghpoor S, et al. Relation between serum ferritin and liver and heart MRI T2* in beta thalassemia major patients. Eastern Mediterranean Health J 2013;19(8):727-32.

[11] Westwood M, Anderson LJ, Firmin DN, et al. A single breath-hold multiecho $\mathrm{T} 2 *$ cardiovascular magnetic resonance technique for diagnosis of myocardial iron overload. J Magn Reson Imaging 2003;18(1):33-9.

\section{Original Research Article}

[12] Ghugre NR, Coates TD, Nelson MD, et al. Mechanisms of tissue-iron relaxivity: nuclear magnetic resonance studies of human liver biopsy specimens. Magn Reson Med 2005;54(5):1185-93.

[13] Noetzli LJ, Carson SM, Nord AS, et al. Longitudinal analysis of heart and liver iron in thalassemia major. Blood 2008;112(7):2973-8.

[14] Kaltwasser JP, Gottschalk R, Schalk KP, et al. Noninvasive quantitation of liver iron overload by magnetic resonance imaging. $\mathrm{Br} \mathrm{J}$ Haematol 1990;74(3):360-3. 